\title{
Searching for the signal of dark matter and photon associated production at the LHC beyond leading order
}

\author{
Fa Peng Huang, ${ }^{1}$ Chong Sheng $\mathrm{Li}^{*},{ }^{1,2}$ Jian Wang, ${ }^{1}$ and Ding Yu Shao ${ }^{1}$ \\ ${ }^{1}$ School of Physics and State Key Laboratory of Nuclear Physics and Technology, \\ Peking University, Beijing, 100871, China \\ ${ }^{2}$ Center for High Energy Physics, Peking University, Beijing, 100871, China
}

\begin{abstract}
We study the signal of dark matter and photon associated production induced by the vector and axial-vector operators at the LHC, including the QCD next-to-leading order (NLO) effects. We find that the QCD NLO corrections reduce the dependence of the total cross sections on the factorization and renormalization scales, and the $K$ factors increase with the increasing of the dark matter mass, which can be as large as about 1.3 for both the vector and axial-vector operators. Using our QCD NLO results, we improve the constraints on the new physics scale from the results of the recent CMS experiment. Moreover, we show the Monte Carlo simulation results for detecting the $\gamma+E_{T}$ signal at the QCD NLO level, and present the integrated luminosity needed for a $5 \sigma$ discovery at the $14 \mathrm{TeV}$ LHC . If the signal is not observed, the lower limit on the new physics scale can be set.

PACS numbers: 12.38.Bx, 14.65.Jk, 14.70.Bh, 95.35.+d
\end{abstract}

\footnotetext{
* Electronic address: csli@pku.edu.cn
} 


\section{INTRODUCTION}

The dark matter (DM) attracts a lot of attention in the fields of both cosmology and

particle physics [1, 2]. The astrophysical observations have provided strong evidence for the existence of DM [3]. Compared to the direct and indirect experiments, the hadron colliders have impressive advantages that the measurements are not sensitive to the uncertainties related to the galactic distributions, DM velocities, etc. There have been a lot of studies to search for DM at the LHC in a series of DM models [4-20]. We can probe the DM through the visible particles, which are associated produced, such as a photon or a jet [21-23]. In this work, we only consider the DM and photon associated production at the LHC, since this signal is clear and suffers from less backgrounds from the standard model (SM).

Recently, the CMS collaboration has searched for new physics (NP) in the $\gamma+\#_{T}$ final state, and set the $90 \%$ confidence level (C.L.) lower limits on the NP scale for vector and axial-vector operators [24]. However, the analysis for the DM searching there is based on the leading order ( $\mathrm{LO}$ ) results, which suffer from large uncertainties due to the choice of renormalization and factorization scales. In our previous work [25], we only considered the QCD next-to-leading order (NLO) corrections for the case of the scalar operator. Following the ideas of our previous works [25, 26], in this paper, we study the signal of DM and photon associated production induced by the vector and axial-vector operators at the LHC, including QCD NLO corrections. Using our NLO results, we improve the constraints on the NP scale from the results of recent CMS experiment.

In Sec. III, we show the vector and axial-vector operators describing the interactions between DM and the SM particles. In Sec. III, we show the constraints on the DM mass and the NP scale from the relic abundance. In Sec. IV, the numerical results are presented and discussed. In Sec. V, we analyze the backgrounds in the SM and discuss the discovery potential at the $14 \mathrm{TeV}$ LHC. Section VI contains a brief conclusion.

\section{VECTOR AND AXIAL-VECTOR OPERATORS}

We consider the dimension six vector and axial-vector operators

$$
\begin{aligned}
\mathcal{O}_{V} & =\frac{\kappa}{\Lambda^{2}}\left(\bar{q} \gamma^{\mu} q\right)\left(\bar{\chi} \gamma_{\mu} \chi\right), \\
\mathcal{O}_{A} & =\frac{\kappa}{\Lambda^{2}}\left(\bar{q} \gamma^{\mu} \gamma_{5} q\right)\left(\bar{\chi} \gamma_{\mu} \gamma_{5} \chi\right),
\end{aligned}
$$




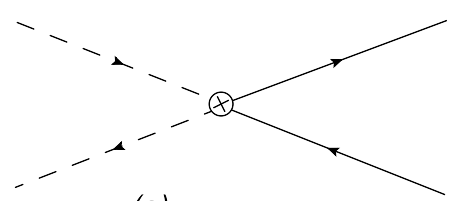

(a)

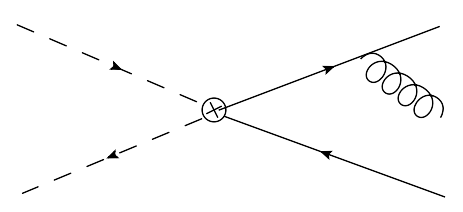

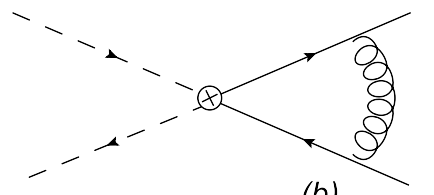

(b)

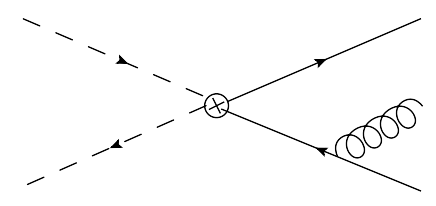

(c)

FIG. 1: Feynman diagrams for the DM annihilation process. Labels (a), (b) and (c) denote the LO, virtual correction and real corrections, respectively.

which are also studied in Refs. [13, 21, 27-29]. The NP scale $\Lambda$ can be regarded as the remnant of integrating the massive propagator between the DM and SM particles. We assume that the Dirac fermion $\chi$ is a DM candidate, and a singlet under the SM gauge group $S U(3)_{c} \times S U(2)_{L} \times U(1)_{Y}$. The DM $\chi$ can only interact with the quarks by these operators. In Ref. [24], the constraints on the NP scale $\Lambda$ for the vector and axial-vector operators are given by the CMS collaboration through the process of DM and photon associated production at LO. In this paper, we will perform the QCD NLO corrections to these processes, whose effects are important for research at the LHC, and improve the limits on the NP scale.

\section{CONSTRAINTS FROM THE RELIC ABUNDANCE}

Before discussing the signal of the DM at the LHC, we first consider the relic abundance which is a precise observable in cosmology. The relic abundance can impose strong constraints on the properties of the DM, and can be obtained from the DM annihilation cross section. The Feynman diagrams are shown in Fig. 1. First, we get the LO annihilation cross section for the vector operator

$$
\sigma_{B, V}^{\mathrm{an}}=N_{c} N_{f} \frac{\kappa^{2}}{\Lambda^{4}} \sqrt{\frac{s}{s-4 m^{2}}} \frac{s+2 m^{2}}{12 \pi},
$$

where $s$ is the square of center-of-mass energy. $N_{c}$ and $N_{f}$ are the numbers of color and flavor of quarks, respectively. $m$ is the mass of the DM. This LO cross section in Eq. (2) is consistent with the unexpanded result in Eq. (8) of Ref. [27]. For the case of vector 
operator, we get the QCD NLO corrections

$$
\sigma_{\mathrm{NLO}, \mathrm{V}}^{a n}=K^{\mathrm{an}} \sigma_{B, V}^{\mathrm{an}}
$$

where $K^{\text {an }}=1+\alpha_{s} / \pi$ is the $K$ factor of the cross section, generally defined as $\sigma_{\mathrm{NLO}} / \sigma_{\mathrm{LO}}$.

We assume that the DM is moving at nonrelativistic velocities $(v \ll 1)$ when freezing out. We define $v$ as the relative velocity between the DM so that the square of the center-of-mass energy can be written as $s \approx 4 m^{2}+m^{2} v^{2}+m^{2} v^{4} / 4$. Thus we can expand

$$
\sigma_{\mathrm{NLO}, \mathrm{V}}^{a n} v \approx a+b v^{2}
$$

where

$$
\begin{aligned}
& a=K^{\mathrm{an}} N_{c} N_{f} \frac{\kappa^{2}}{\Lambda^{4}} \frac{m^{2}}{\pi} \\
& b=K^{\mathrm{an}} N_{c} N_{f} \frac{\kappa^{2}}{\Lambda^{4}} \frac{m^{2}}{6 \pi}
\end{aligned}
$$

For the case of axial-vector operator, we follow the same process and give only the main results. The LO cross section is

$$
\sigma_{B, A}^{\mathrm{an}}=N_{c} N_{f} \frac{\kappa^{2}}{\Lambda^{4}} \sqrt{\frac{s}{s-4 m^{2}}} \frac{s-4 m^{2}}{12 \pi},
$$

which is consistent with the unexpanded result in Eq. (9) of Ref. [27]. After including the QCD NLO corrections to the process induced by the axial-vector operator, we get

$$
\sigma_{\mathrm{NLO}, \mathrm{A}}^{\mathrm{an}} v \approx a+b v^{2}
$$

where

$$
\begin{aligned}
a & =0, \\
b & =\left(1+\frac{\alpha_{s}}{\pi}\right) N_{c} N_{f} \frac{\kappa^{2}}{\Lambda^{4}} \frac{m^{2}}{6 \pi} .
\end{aligned}
$$

Our expanded results in Eqs. (5) and (8) are consistent with the results in Refs. [21, 30].

We perform the calculation of relic abundance by using the method in Ref. [31]. Then we show the constraints on the NP scale and DM mass in Fig. 2. We see that the NLO corrections increase the lower limits on the NP scale slightly. The regions below the red band are allowed, since we assume that this kind of DM is not the unique candidate. 

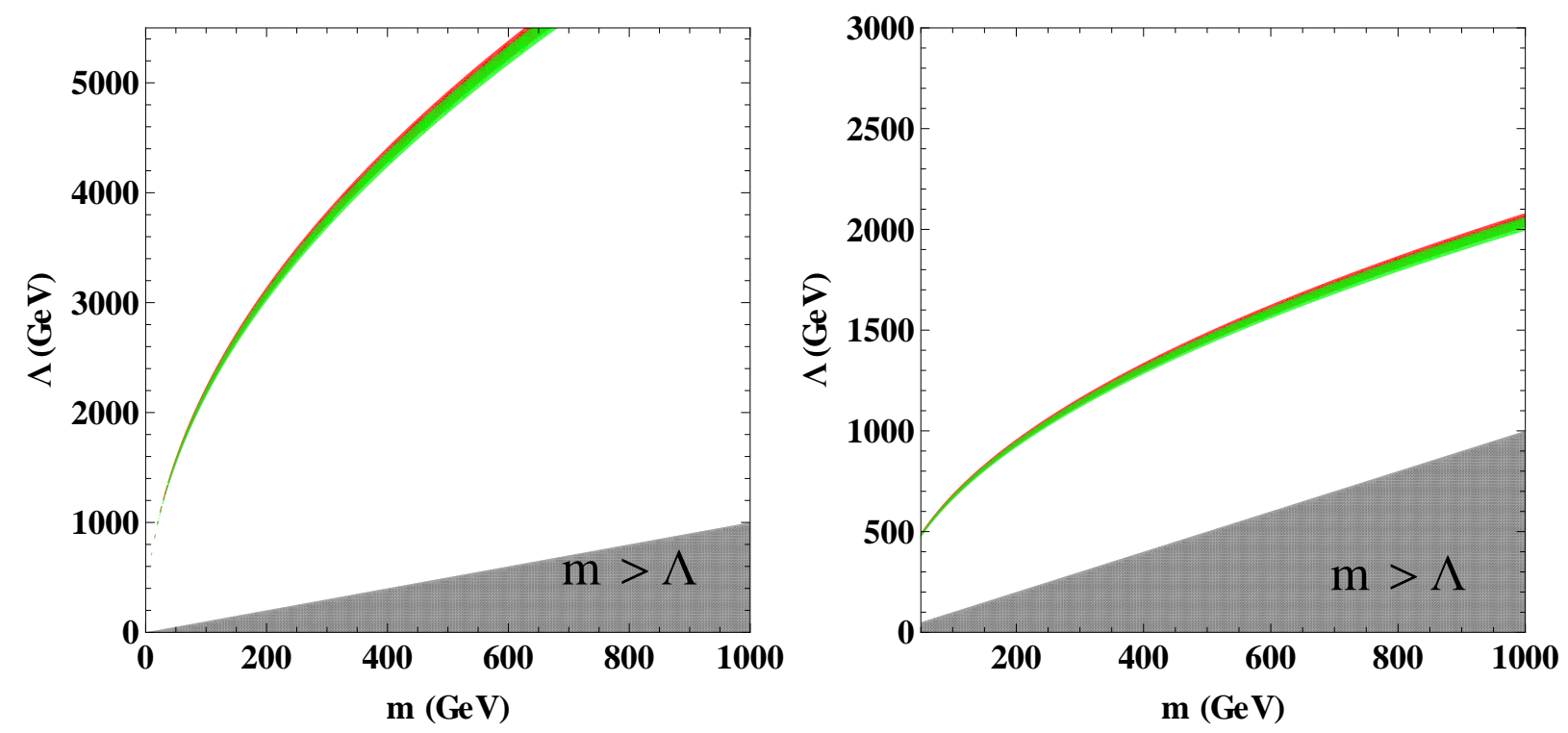

FIG. 2: Constraints on the DM mass and the NP scale for the vector (left) and axial-vector (right) operators, respectively . The relic abundance is required to be in the $2 \sigma$ region around the observed central value [32]. The lower green band is the LO result. The upper red band is the NLO result. In this figure, we choose $\kappa=1, \alpha_{s}=0.118$ and $N_{f}=5$.

\section{NUMERICAL RESULTS OF THE QCD NLO CORRECTIONS FOR THE CASE OF THE VECTOR AND AXIAL-VECTOR OPERATORS}

Different from the scalar operator in our previous work [25], the quark sector and the DM sector can not factorize for the vector and axial-vector operators that we consider in this paper. This leads to more complicated analytical expressions in our calculation. We follow the same approach in our previous paper [25] to cancel the infrared (IR) divergences in QCD NLO calculations, and show the numerical results for the case of vector and axialvector operators below.

\section{A. QCD corrections for the case of the vector and the axial-vector operators}

First of all, we calculate the LO cross section of the following process

$$
q\left(p_{1}\right)+\bar{q}\left(p_{2}\right) \rightarrow \chi\left(p_{3}\right)+\bar{\chi}\left(p_{4}\right)+\gamma\left(p_{5}\right)
$$

The LO Feynman diagrams are shown in Fig. 3. The LO partonic cross section is 

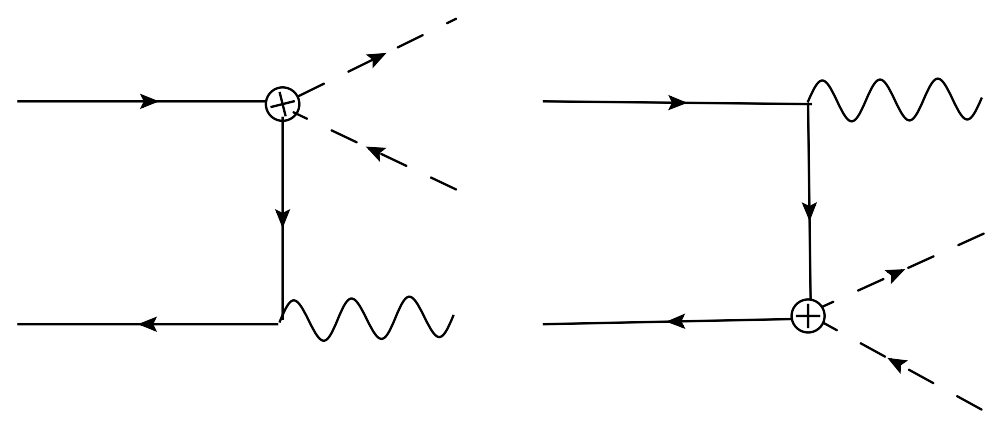

FIG. 3: LO Feynman diagrams for the DM and photon associated production.

$$
\hat{\sigma}_{B}=\frac{1}{2 s_{12}} \int d \Gamma_{3} \overline{\left|\mathcal{M}_{B}\right|^{2}}
$$

where $\Gamma_{3}$ is the three-particle final states phase space. We define $s_{i j}=\left(p_{i}+p_{j}\right)^{2}, t_{i j}=$ $\left(p_{i}-p_{j}\right)^{2}$ and $\alpha=e^{2} / 4 \pi$. The spin and color summed and averaged Born matrix element squared is

$$
\overline{\left|\mathcal{M}_{B}\right|^{2}}=\frac{\pi \alpha \kappa^{2}}{3 \Lambda^{4}} \sum_{i} Q_{i}^{2}\left|\mathcal{M}_{0}^{V(A)}\right|^{2},
$$

where $\left|\mathcal{M}_{0}^{V}\right|^{2}$ for the case of the vector operator is expressed as

$$
\begin{aligned}
\left|\mathcal{M}_{0}^{V}\right|^{2}= & \frac{1}{t_{15} t_{25}} 16\left(2 m^{4}\left(4 s_{12}+t_{15}+t_{25}\right)+m^{2}\left(-s_{12}\left(2 s_{35}\right.\right.\right. \\
& \left.+2 s_{45}+3 t_{13}+3 t_{14}-4 t_{15}+3 t_{23}+3 t_{24}-4 t_{25}\right) \\
& -s_{45} t_{15}-s_{45} t_{25}-s_{35}\left(t_{15}+t_{25}\right)+ \\
& 4\left(s_{12}\right)^{2}+2\left(t_{15}\right)^{2}+2\left(t_{25}\right)^{2}-2 t_{13} t_{15}-2 t_{14} t_{15}+t_{15} t_{23}+t_{15} t_{24}+ \\
& \left.t_{13} t_{25}+t_{14} t_{25}-2 t_{23} t_{25}-2 t_{24} t_{25}\right)+s_{45} t_{13} t_{15}+ \\
& s_{35} t_{14} t_{15}+s_{12}\left(s_{45}\left(t_{13}+t_{23}\right)+\right. \\
& \left.s_{35}\left(t_{14}+t_{24}\right)+2\left(t_{14} t_{23}+t_{13} t_{24}\right)\right)+s_{45} t_{23} t_{25}+s_{35} t_{24} t_{25}+ \\
& t_{14} t_{15} t_{23}+t_{13} t_{15} t_{24}-2 t_{15} t_{23} t_{24}-2 t_{13} t_{14} t_{25}+ \\
& \left.t_{14} t_{23} t_{25}+t_{13} t_{24} t_{25}\right),
\end{aligned}
$$

and $Q_{i}(i=1,5)$ are the electric charge of the quarks. For the case of axial-vector operator,

$$
\left|\mathcal{M}_{0}^{A}\right|^{2}=\left|\mathcal{M}_{0}^{V}\right|^{2}-\frac{64 m^{2}\left(2 s_{12}\left(t_{15}+t_{25}\right)+2\left(s_{12}\right)^{2}+\left(t_{15}\right)^{2}+\left(t_{25}\right)^{2}\right)}{t_{15} t_{25}} .
$$

The LO total cross section at the LHC is obtained by convoluting the partonic cross section with the parton distribution functions $(\mathrm{PDFs}) G_{q(\bar{q})}(x)$ :

$$
\sigma_{B}=\int d x_{1} d x_{2}\left[G_{q / p}\left(x_{1}\right) G_{\bar{q} / p}\left(x_{2}\right)+\left(x_{1} \leftrightarrow x_{2}\right)\right] \hat{\sigma}_{B} .
$$




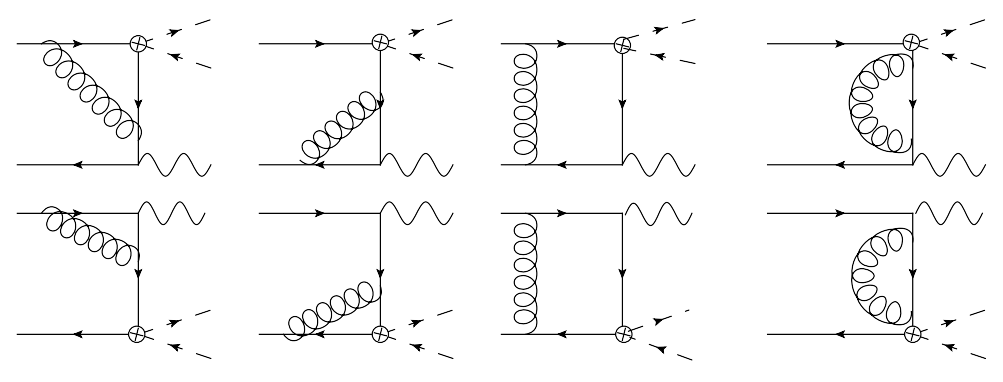

FIG. 4: Feynman diagrams for one-loop virtual corrections.
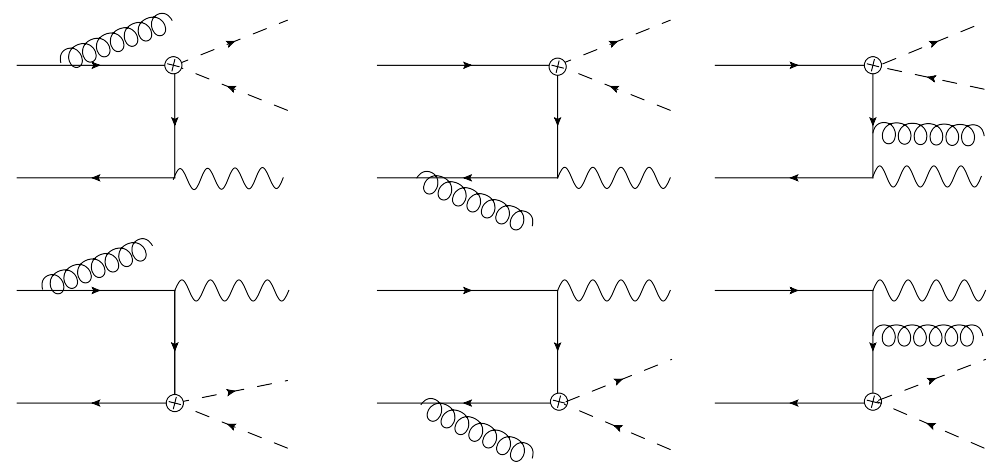

FIG. 5: Feynman diagrams for a real gluon emission.

The QCD NLO corrections consist of real gluon radiation, quark or antiquark emission and one-loop virtual gluon effects. We use dimensional regularization to regulate both the ultraviolet (UV) and the IR divergences in our calculations.

After renormalization, the UV divergences in the virtual corrections are removed, leaving the IR divergences and the finite terms. The final virtual gluon corrections to the partonic cross section are

$$
\hat{\sigma}_{v}=\frac{1}{2 s_{12}} \int d \Gamma_{3} 2 \overline{\operatorname{Re}\left(\mathcal{M}_{B}^{*} \mathcal{M}_{v}\right)}
$$

for which the Feynman diagrams are shown in Fig. 4. The IR divergent part of $\mathcal{M}_{v}$ is given by

$$
\mathcal{M}_{v}^{I R}=\frac{\alpha_{s}}{4 \pi} C_{\epsilon}\left(\frac{A_{2}^{v}}{\epsilon^{2}}+\frac{A_{1}^{v}}{\epsilon^{1}}\right) \mathcal{M}_{B},
$$

where $C_{\epsilon}=\Gamma(1+\epsilon)\left[\left(4 \pi \mu_{R}^{2}\right) / s_{12}\right]^{\epsilon}$ and

$$
\begin{aligned}
& A_{2}^{v}=-2 C_{F}, \\
& A_{1}^{v}=-3 C_{F} .
\end{aligned}
$$

The Feynman diagrams for the real gluon radiation process

$$
q\left(p_{1}\right)+\bar{q}\left(p_{2}\right) \rightarrow \chi\left(p_{3}\right)+\bar{\chi}\left(p_{4}\right)+\gamma\left(p_{5}\right)+g\left(p_{6}\right)
$$


are shown in Fig. 5, Soft and collinear divergences appear when we perform the final state phase integrations. To cancel the IR singularities, we use the two cutoff phase space slicing method to integrate the singular regions analytically [33]. Explicitly, we use the soft cutoff parameter $\delta_{s}$ to define the soft regions and the collinear cutoff parameter $\delta_{c}$ to define the hard collinear regions. The soft regions are just the phase space where the real radiated gluon's energy $E_{6} \leq \delta_{s} \sqrt{s_{12}} / 2$. The collinear regions are defined by $\left|t_{i 6}\right|<\delta_{c} s_{12}$ with $i=1,2$. Thus, the partonic cross section of the real gluon radiation can be separated as

$$
d \hat{\sigma}_{r}=d \hat{\sigma}_{r}^{S}+d \hat{\sigma}_{r}^{H C}+d \hat{\sigma}_{r}^{\overline{H C}}
$$

Here, $\hat{\sigma}_{r}^{S}$ and $\hat{\sigma}_{r}^{H C}$ represent the partonic cross section for the soft regions and hard collinear regions, respectively. The hard noncollinear part $\hat{\sigma}_{r}^{\overline{H C}}$ is finite and can be computed numerically using standard Monte Carlo integration techniques.

In the soft regions, using the eikonal approximation, the cross section can be factorized as

$$
d \hat{\sigma}_{r}^{S}=d \hat{\sigma}_{B} \frac{\alpha_{s}}{2 \pi} C_{\epsilon}\left(\frac{A_{2}^{S}}{\epsilon^{2}}+\frac{A_{1}^{S}}{\epsilon}+A_{0}^{S}\right)
$$

where

$$
A_{2}^{S}=2 C_{F}, \quad A_{1}^{S}=-4 C_{F} \ln \delta_{s}
$$

In the hard collinear limits, the squared matrix element factors into the product of a splitting kernel and a leading order squared matrix element. Then we obtain

$$
\begin{aligned}
d \sigma_{r}^{H C} & =d \hat{\sigma}_{B} \frac{\alpha_{s}}{2 \pi} C_{\epsilon}\left(-\frac{1}{\epsilon}\right) \delta_{c}^{-\epsilon}\left[P_{q q}(z, \epsilon) G_{q / p}\left(x_{1} / z\right) G_{\bar{q} / p}\left(x_{2}\right)\right. \\
& \left.+P_{\bar{q} \bar{q}}(z, \epsilon) G_{\bar{q} / p}\left(x_{1}\right) G_{q / p}\left(x_{2} / z\right)+\left(x_{1} \leftrightarrow x_{2}\right)\right] \frac{d z}{z}\left(\frac{1-z}{z}\right)^{-\epsilon} d x_{1} d x_{2}
\end{aligned}
$$

in which the $P_{i j}(z, \epsilon)$ are the unregulated splitting function. To factorize the collinear singularity into the PDFs, we use scale dependent PDFs in the $\overline{\mathrm{MS}}$ convention:

$$
G_{b / p}\left(x, \mu_{F}\right)=G_{b / p}(x)+\left(-\frac{1}{\epsilon}\right)\left[\frac{\alpha_{s}}{2 \pi} \frac{\Gamma(1-\epsilon)}{\Gamma(1-2 \epsilon)}\left(\frac{4 \pi \mu_{R}^{2}}{\mu_{F}^{2}}\right)^{\epsilon}\right] \int_{x}^{1} \frac{d z}{z} P_{b a}(z) G_{a / p}(x / z) .
$$

Now, we replace $G_{q(\bar{q}) / p}$ in the LO hadronic cross section (14) and combine the result with the hard collinear contribution in Eq. (22). The resulting $\mathcal{O}\left(\alpha_{s}\right)$ expression for the initial 

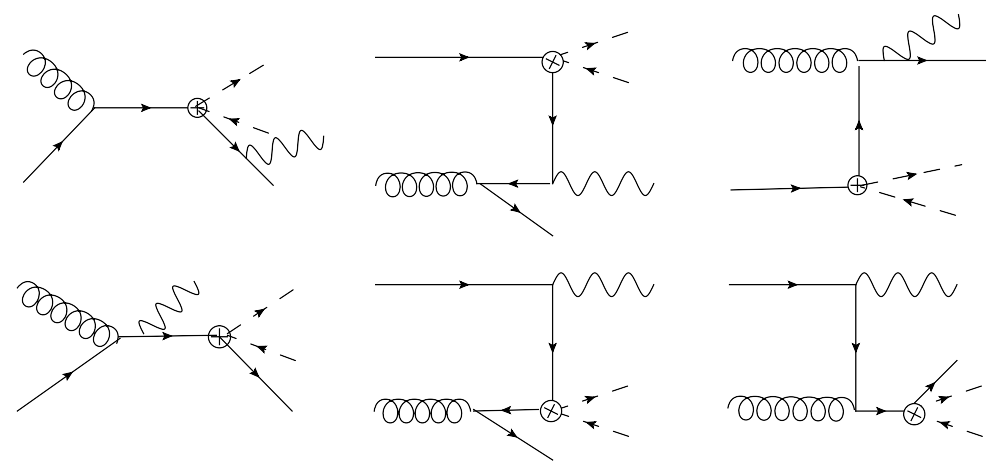

FIG. 6: Feynman diagrams for a quark emission. The Feynman diagrams for antiquark emission can be obtained by charge conjugation.

state collinear contribution is

$$
\begin{aligned}
d \sigma^{\text {coll }} & =d \hat{\sigma}_{B} \frac{\alpha_{s}}{2 \pi} C_{\epsilon}\left\{\tilde{G}_{q / p}\left(x_{1}, \mu_{F}\right) G_{\bar{q} / p}\left(x_{2}, \mu_{F}\right)+G_{q / p}\left(x_{1}, \mu_{F}\right) \tilde{G}_{\bar{q} / p}\left(x_{2}, \mu_{F}\right)\right. \\
& +\sum_{a=q, \bar{q}}\left[\frac{A_{1}^{s c}(a \rightarrow a g)}{\epsilon}+A_{0}^{s c}(a \rightarrow a g)\right] G_{q / p}\left(x_{1}, \mu_{F}\right) G_{\bar{q} / p}\left(x_{2}, \mu_{F}\right) \\
& \left.+\left(x_{1} \leftrightarrow x_{2}\right)\right\} d x_{1} d x_{2} .
\end{aligned}
$$

with

$$
A_{1}^{s c}(q \rightarrow q g)=C_{F}\left(2 \ln \delta_{s}+3 / 2\right) .
$$

The $\tilde{G}$ functions are given by

$$
\tilde{G}_{b / p}\left(x, \mu_{F}\right)=\sum_{a} \int_{x}^{1-\delta_{s} \delta_{a b}} \frac{d y}{y} G_{a / p}\left(x / y, \mu_{F}\right) \tilde{P}_{b a}(y)
$$

with

$$
\tilde{P}_{b a}(y)=P_{b a}(y) \ln \left(\delta_{c} \frac{1-y}{y} \frac{s_{12}}{\mu_{F}^{2}}\right)-P_{b a}^{\prime}(y) .
$$

A complete real correction includes also the (anti)quark emitted processes, as shown in Fig. 6, such as

$$
g\left(p_{1}\right)+q / \bar{q}\left(p_{2}\right) \rightarrow \chi\left(p_{3}\right)+\bar{\chi}\left(p_{4}\right)+\gamma\left(p_{5}\right)+q / \bar{q}\left(p_{6}\right) .
$$

We only need to deal with the collinear divergences which can be totally absorbed into the redefinition of the PDFs in Eq. (23) for these processes.

Finally, the NLO total cross section for the process $p p \rightarrow \chi \bar{\chi} \gamma$ is

$$
\begin{aligned}
\sigma^{N L O} & =\int d x_{1} d x_{2}\left\{\left[G_{q / p}\left(x_{1}, \mu_{F}\right) G_{\bar{q} / p}\left(x_{2}, \mu_{F}\right)+\left(x_{1} \leftrightarrow x_{2}\right)\right]\left(\hat{\sigma}_{B}+\hat{\sigma}_{v}+\hat{\sigma}_{r}^{S}+\hat{\sigma}_{r}^{\overline{H C}}\right)\right\}+\sigma^{\text {coll }} \\
& +\sum_{a=q, \bar{q}} \int d x_{1} d x_{2}\left[G_{g / p}\left(x_{1}, \mu_{F}\right) G_{a / p}\left(x_{2}, \mu_{F}\right)+\left(x_{1} \leftrightarrow x_{2}\right)\right] \hat{\sigma}_{r} \bar{C}(g a \rightarrow \chi \bar{\chi} \gamma a),
\end{aligned}
$$


where $\bar{C}$ in $\hat{\sigma}_{r}{ }^{\bar{C}}(g a \rightarrow \chi \bar{\chi} \gamma a)$ means that the phase space integration is performed in the noncollinear regions. Note that the above expression contains no singularities since

$$
A_{2}^{v}+A_{2}^{S}=0, \quad A_{1}^{v}+A_{1}^{S}+2 A_{1}^{s c}(q \rightarrow q g)=0
$$

and we can perform numerical integration now.

\section{B. Numerical results}

We use the CTEQ6L1 (CTEQ6M) PDF sets [34] and the corresponding strong coupling $\alpha_{s}$ for the LO (NLO) calculations. The default factorization scale $\mu_{F}$ and renormalization scale $\mu_{R}$ are set as $2 m$. Recently, the observations of the gamma ray in Fermi-LAT give the hints of $130 \mathrm{GeV}$ DM [35, 36]. Thus, we set the default parameters $(m, \Lambda)=(130 \mathrm{GeV}, 500 \mathrm{GeV})$

and $\kappa=1$ unless otherwise specified, which are allowed by the constraints of relic abundance. Here, we choose the kinematic cuts

$$
\begin{aligned}
p_{T}^{\gamma} & >100 \mathrm{GeV} \\
\left|\eta^{\gamma}\right| & <2.4 \\
p_{T}^{\text {miss }} & >100 \mathrm{GeV} \\
p_{T}^{j e t} & >20 \mathrm{GeV} \\
\left|\eta^{j e t}\right| & <2.5 \\
\sum_{R_{j \gamma} \in R_{0}} p_{T}^{j e t} & <p_{T}^{\gamma}\left(\frac{1-\cos R_{j \gamma}}{1-\cos R_{0}}\right),
\end{aligned}
$$

where $R \equiv \sqrt{\Delta \phi^{2}+\Delta \eta^{2}}$ and $R_{0}=0.4$.

In Figs. 7 and 8, we show the dependence of the LO (NLO) cross sections for the DM and photon associated production at the LHC on the factorization scale $\mu_{F}$ and renormalization scale $\mu_{R}$. It can be seen that the dependence of the NLO cross section on the factorization scale $\mu_{F}$ and renormalization scale $\mu_{R}$ is significantly reduced, compared to the LO cross section. This makes the theoretical prediction much more reliable.

In Fig. 9, we show the DM mass dependence of the LO and NLO cross sections for producing heavy DM at the $14 \mathrm{TeV}$ LHC induced by the vector operator. When the DM mass varies from 130 to $200 \mathrm{GeV}$, the QCD NLO corrections are modest. For the DM mass in the range from 300 to $1000 \mathrm{GeV}$, the QCD NLO corrections generally improve the cross 

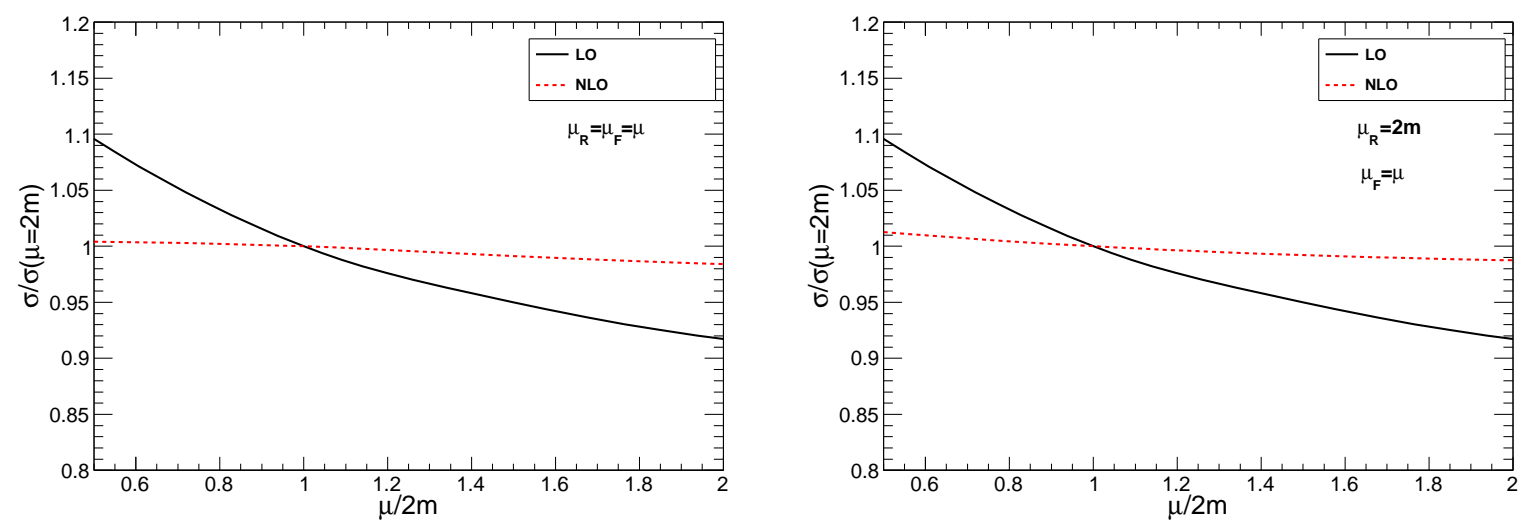

FIG. 7: Dependence of the LO (NLO) cross sections on the factorization scale $\mu_{F}$ and renormalization scale $\mu_{R}$ for the vector operator.
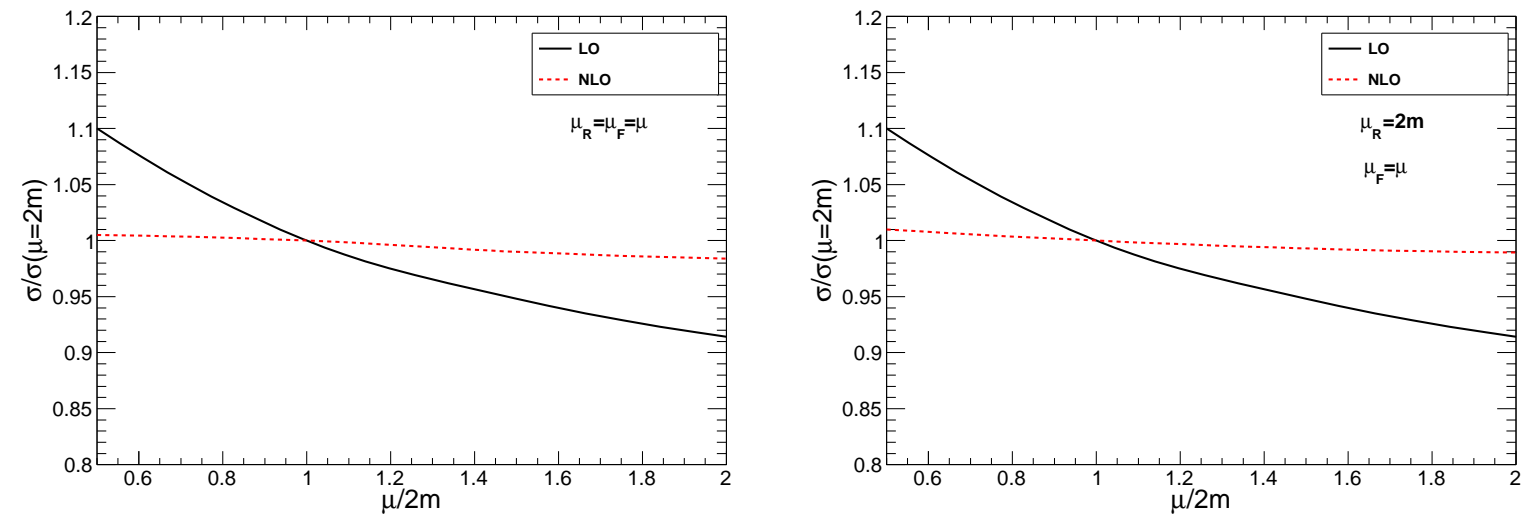

FIG. 8: Dependence of the LO (NLO) cross sections on the factorization scale $\mu_{F}$ and renormalization scale $\mu_{R}$ for the axial-vector operator.

section and are more significant for larger DM mass. For example, the QCD NLO corrections increase the cross sections by about $19 \%$ for $m=1000 \mathrm{GeV}$. Thus, it is necessary to consider the NLO corrections to the process of DM production at hadron colliders. We also show the mass dependence of the $K$-factors for the axial-vector operator in Fig. 10, which is similar to the case of vector operator. Since there is no explicit limit on the DM mass, for completeness, we show the NLO results on DM mass from $0.1 \mathrm{GeV}$ to $1000 \mathrm{GeV}$ in Fig. 11, It can be seen that the $K$ factor in the light DM region, i.e. less than $100 \mathrm{GeV}$, is nearly a constant, which is about 0.96 and 0.98 for the case of vector and axial-vector operators, respectively. 

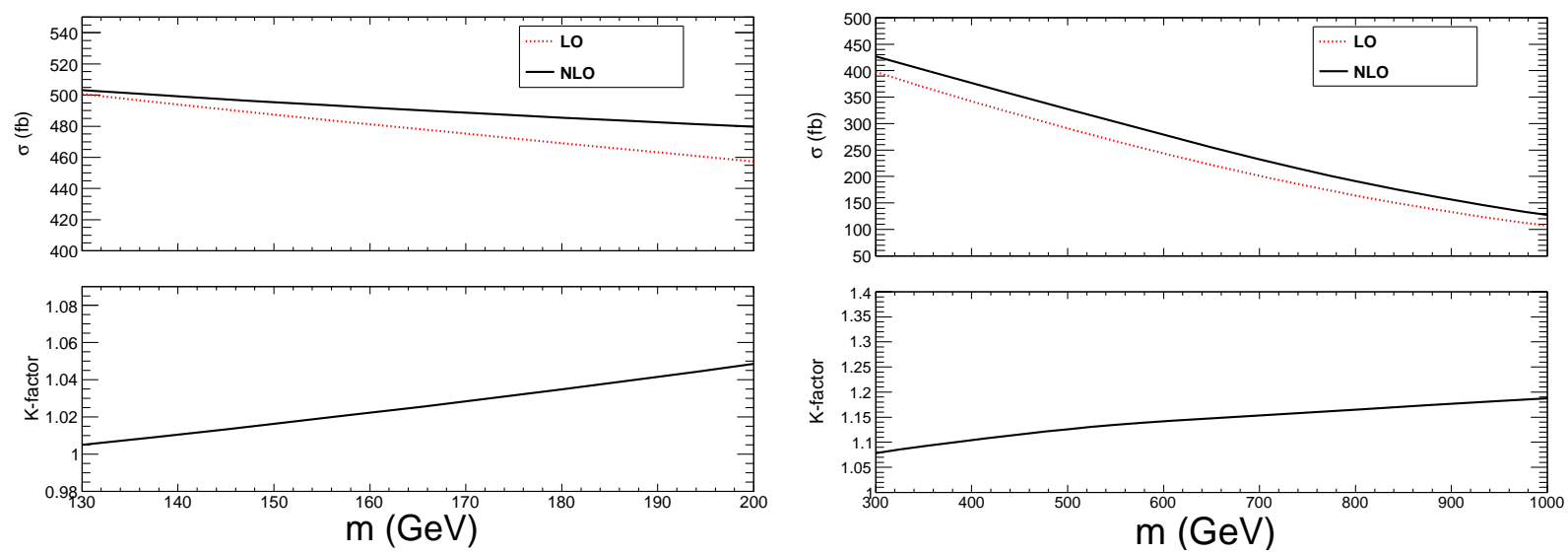

FIG. 9: Dependence of the LO and NLO cross sections on the DM mass for the vector operator at $14 \mathrm{TeV}$ LHC. The $K$ factors are also shown.

In order to compare with the experimental results of CMS, we use the same kinematic cuts and center-of-mass energy as in 24], and improve the lower limits on the NP scale in the results of the CMS collaboration [24], using our $K$ factors at the $7 \mathrm{TeV}$ LHC. Here, we show the improved limits on the NP scale $\Lambda$ in Table $\llbracket$ and $\amalg$ for the vector and axial-vector operators, respectively.
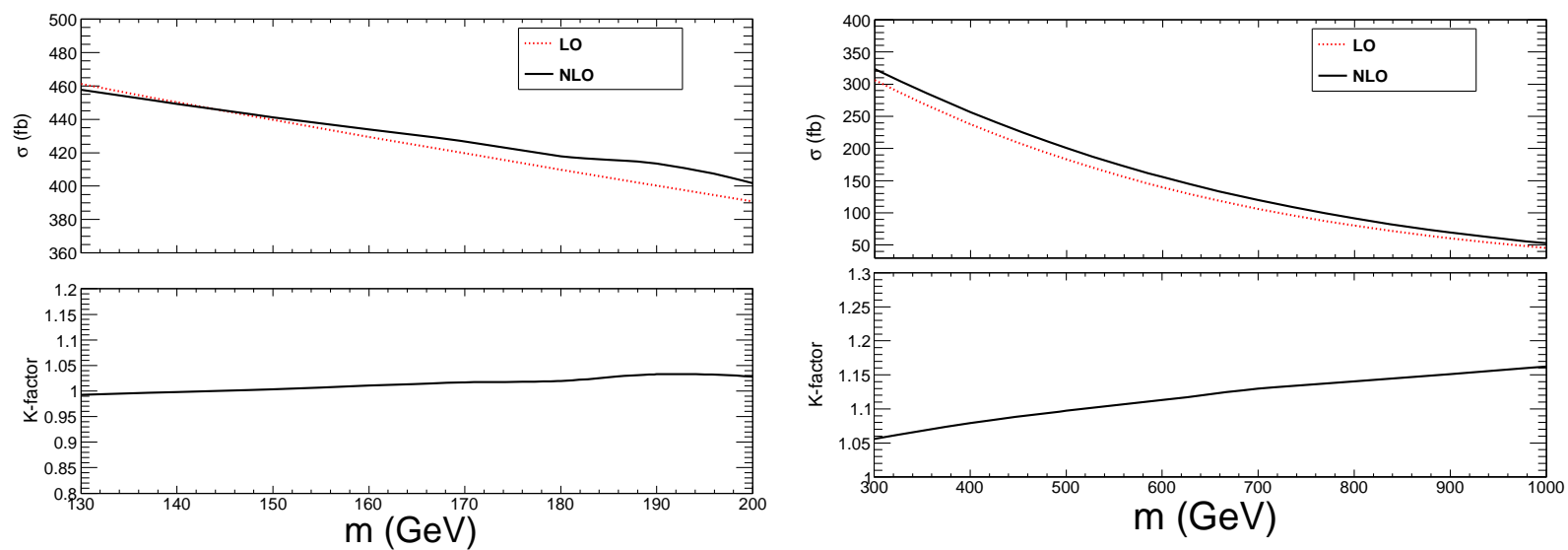

FIG. 10: Dependence of the LO and NLO cross sections on the DM mass for the axial-vector operator at the $14 \mathrm{TeV}$ LHC. The $K$ factors are also shown. 

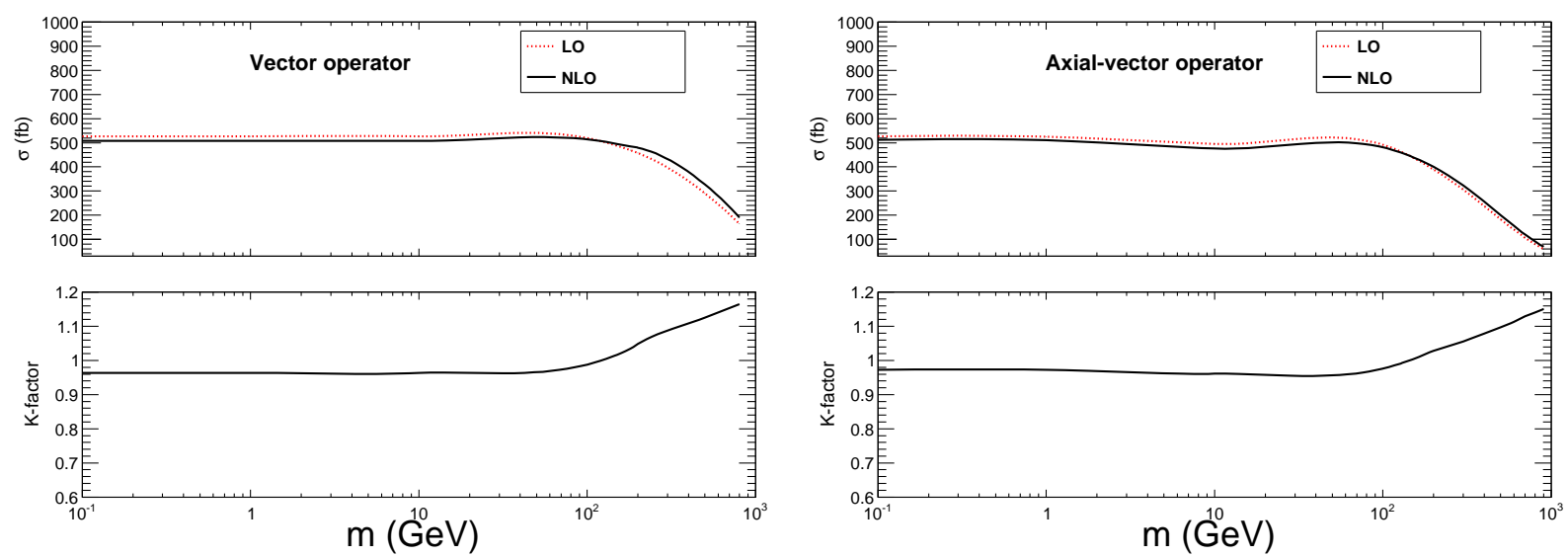

FIG. 11: Dependence of the LO cross section, NLO cross section and $K$ factors on the DM mass at the $14 \mathrm{TeV}$ LHC.

TABLE I: Sample results of the 90\% C.L. lower limits on the NP scale $\Lambda$ for the vector operator. The LO results are given in the CMS analysis [24]. The $K$ factors at the $7 \mathrm{TeV}$ LHC for different DM masses are also shown.

\begin{tabular}{cccc}
\hline \hline$m[\mathrm{GeV}]$ & $\Lambda[\mathrm{GeV}](\mathrm{LO})[24]$ & $\Lambda[\mathrm{GeV}](\mathrm{NLO})$ & $K$ \\
\hline 200 & 549 & 564 & 1.11 \\
500 & 442 & 463 & 1.20 \\
1000 & 246 & 263 & 1.31 \\
\hline \hline
\end{tabular}

TABLE II: Sample results of the $90 \%$ C.L. lower limits on the NP scale $\Lambda$ for the axial-vector operator. The LO results are given in the CMS analysis [24]. The $K$ factors at the $7 \mathrm{TeV}$ LHC for different DM masses are also shown.

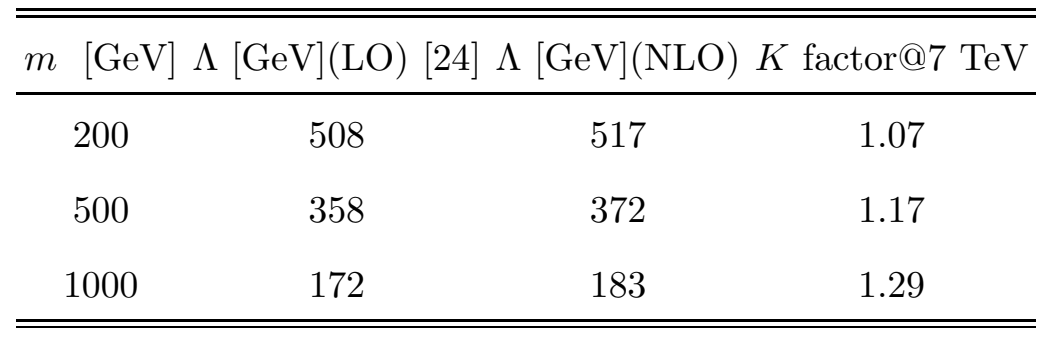

\section{DISCOVERY POTENTIAL}

In this section, we present the Monte Carlo simulation results for detecting the $\gamma+E_{T}$ signal at the $14 \mathrm{TeV}$ LHC with NLO accuracy in perturbative QCD. The main irreducible SM 


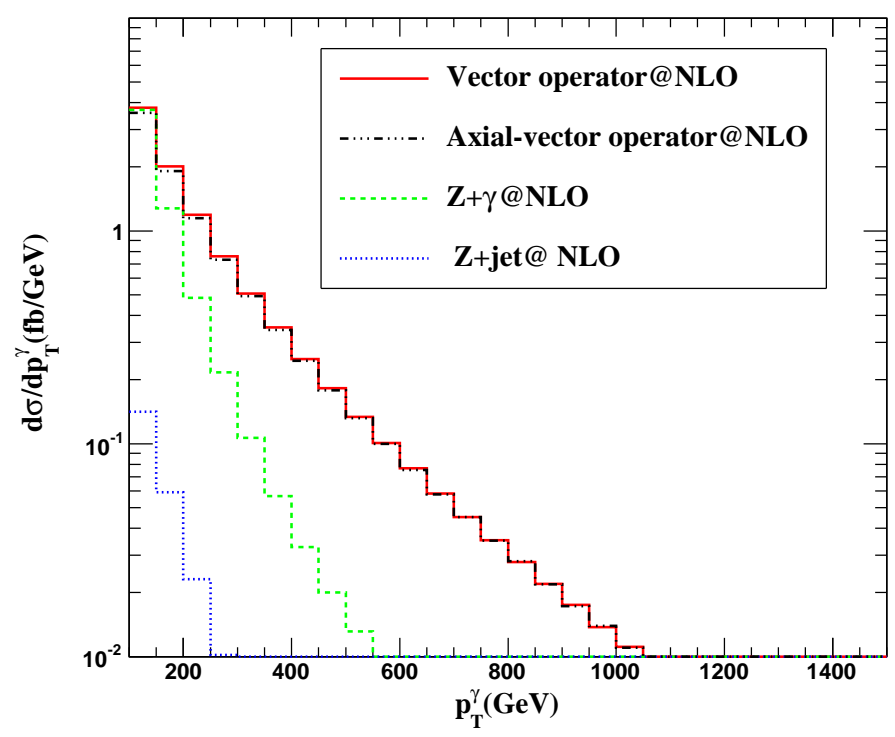

FIG. 12: Dependence of the differential cross section on $p_{T}^{\gamma}$.

backgrounds are the $p p \rightarrow Z(\rightarrow \nu \bar{\nu})+\gamma$ and $p p \rightarrow Z(\rightarrow \nu \bar{\nu})+j$ when the jet is misidentified as a photon. The misidentified probability is set to be $P_{\gamma / j}=10^{-4}$, as pointed out in Ref. [37]. We use the Monte Carlo program MCFM [38-41] to calculate the backgrounds at the NLO level.

Figure 12 and 13 show the differential cross sections as functions of $p_{T}^{\gamma}$ and $p_{T}^{\text {miss }}$, respectively, for the signal and backgrounds at the NLO level. We can see that the differential cross sections of the backgrounds decrease faster than that of the signal as the transverse momentum increases. Thus, the ratio of signal and background can be improved if we set a larger $p_{T}$ cut.

Figure 14 shows the differential cross sections as a function of $\eta^{\gamma}$ for the signal and the backgrounds at the NLO level. We see that the distribution of the signal is more concentrated in the central region than the backgrounds. These distributions give some clues to suppress the backgrounds more efficiently at the LHC.

Figure 15 presents the integrated luminosity needed to discover the signal at a $5 \sigma$ level at the $14 \mathrm{TeV}$ LHC. We find that the needed integrated luminosity grows with the increasing of the NP scale, and depends more strongly on the DM mass for larger NP scale. In particular, for $\Lambda=1000 \mathrm{GeV}$ and $m=200 \mathrm{GeV}$, the needed integrated luminosity is $12 \mathrm{fb}^{-1}$ at the 14 TeV LHC. Figure 16] shows the results for the axial-vector operator. In Fig. 17, we present 


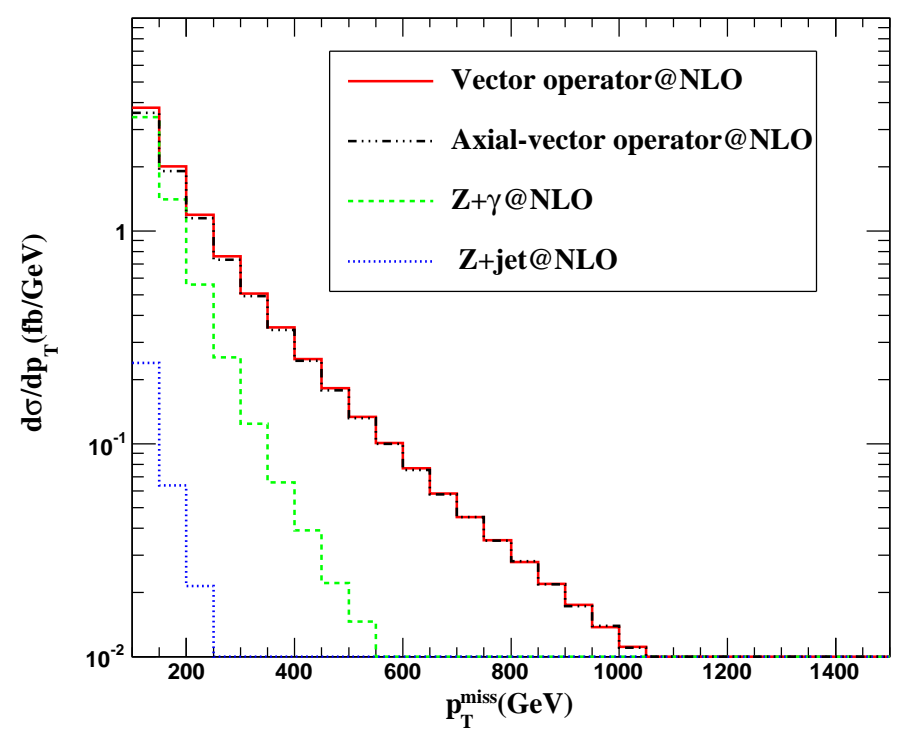

FIG. 13: Dependence of the differential cross section on $p_{\mathrm{T}}^{\text {miss }}$.

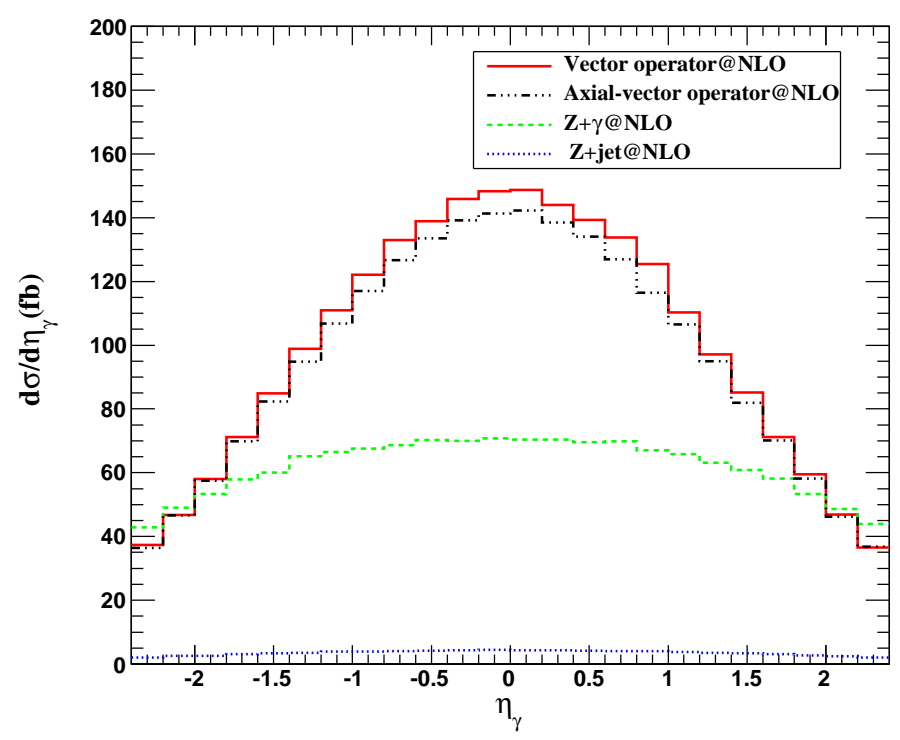

FIG. 14: Dependence of the differential cross section on $\eta^{\gamma}$.

the limits of the NP scale for $3 \sigma$ and $5 \sigma$ exclusions at the $14 \mathrm{TeV}$ LHC, assuming $m=130$ $\mathrm{GeV}$. We see that the NP scale is constrained to be larger than $1200 \mathrm{GeV}$ if the $14 \mathrm{TeV}$ LHC does not detect this signal after collecting an integrated luminosity of $10 \mathrm{fb}^{-1}$. Figure 18 gives the results for the axial-vector operator. 


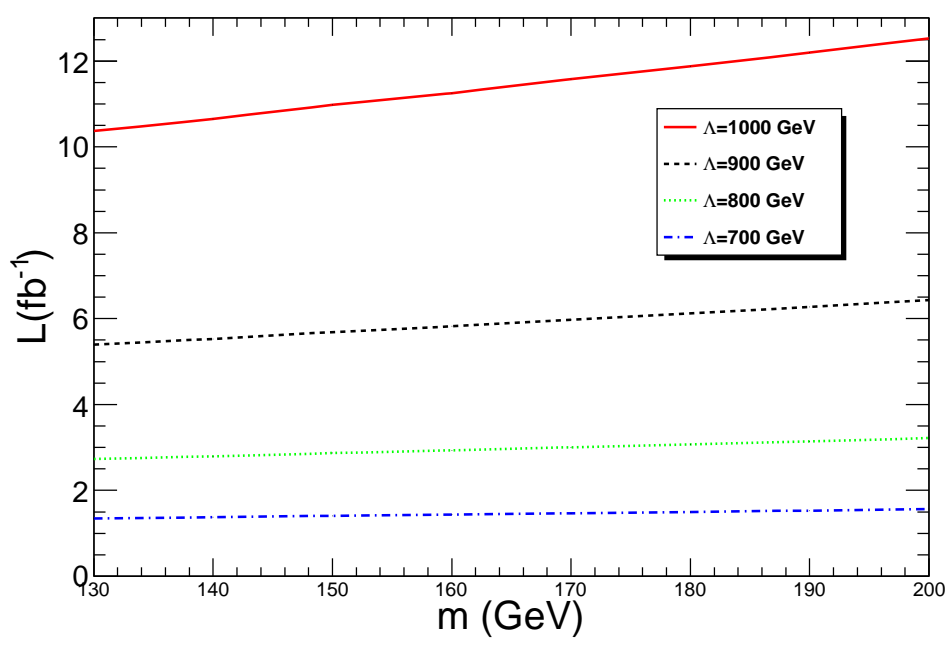

FIG. 15: The integrated luminosity needed for a $5 \sigma$ discovery as a function of the DM mass at the $14 \mathrm{TeV}$ LHC for the vector operator. We choose the cuts $p_{T}^{\gamma}>300 \mathrm{GeV}$ and $p_{T}^{m i s s}>300 \mathrm{GeV}$ due to the above analysis of Fig. 12 and Fig. 13 .

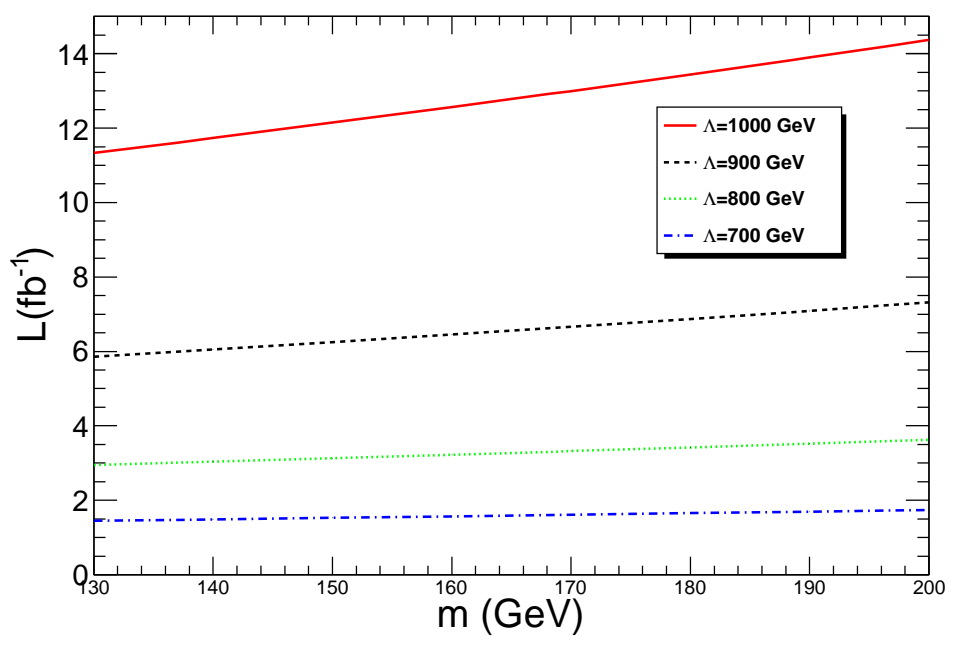

FIG. 16: The integrated luminosity needed for a $5 \sigma$ discovery as a function of the DM mass at the $14 \mathrm{TeV}$ LHC for the axial-vector operator. We choose the cuts $p_{T}^{\gamma}>300 \mathrm{GeV}$ and $p_{T}^{\text {miss }}>300 \mathrm{GeV}$ due to the above analysis of Figs. 12 and 13 ,

\section{CONCLUSION}

We have investigated the signal of DM and photon associated production induced by the vector and axial-vector operators at the LHC, including the QCD NLO effects. We find that 


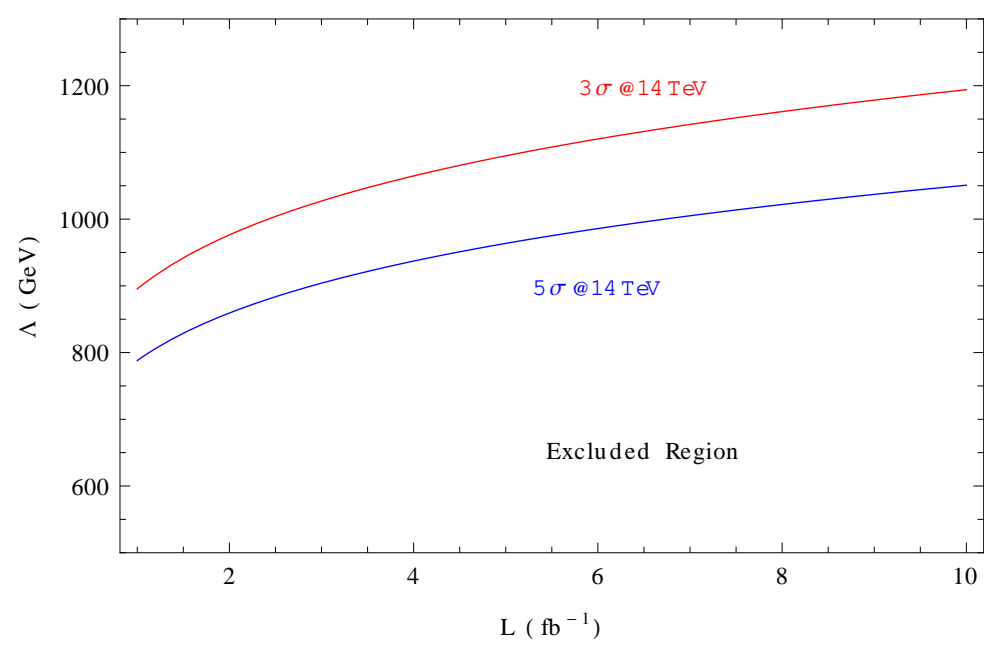

FIG. 17: The limits of the NP scale for $3 \sigma$ and $5 \sigma$ exclusions at the $14 \mathrm{TeV}$ LHC, assuming $m=130$ $\mathrm{GeV}$ for the vector operator. We choose the cuts $p_{T}^{\gamma}>300 \mathrm{GeV}$ and $p_{T}^{\text {miss }}>300 \mathrm{GeV}$ due to the above analysis of Figs. 12 and 13 .

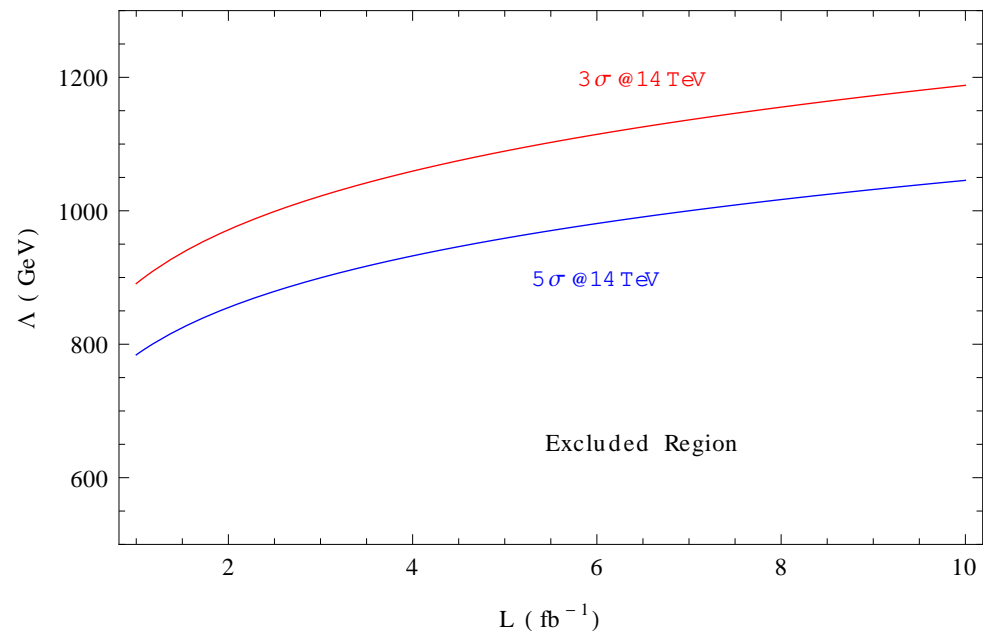

FIG. 18: The limits of the NP scale for $3 \sigma$ and $5 \sigma$ exclusions at the $14 \mathrm{TeV}$ LHC, assuming $m=130$ $\mathrm{GeV}$ for the axial-vector operator. We choose the cuts $p_{T}^{\gamma}>300 \mathrm{GeV}$ and $p_{T}^{\text {miss }}>300 \mathrm{GeV}$ due to the above analysis of Figs. 12 and 13 .

the QCD NLO corrections significantly reduce the dependence of the total cross sections on the factorization and renormalization scales, and the QCD NLO corrections are more significant for larger DM mass for both the vector and axial-vector operators. Using our NLO results, we improve the constraints on the NP scale from the results of the recent CMS experiment. Moreover, we calculate the dominant SM backgrounds at the NLO level, and 
show the differential cross sections of both the signal and backgrounds as functions of $p_{\mathrm{T}}^{\gamma}$,

$p_{\mathrm{T}}^{\text {miss }}$ and $\eta^{\gamma}$. The character of these distributions can help to choose the kinematic cuts in the experiments. Finally, we show the potential to discover the DM at the $14 \mathrm{TeV}$ LHC, and provide the exclusion limits on the NP scale if this signal is not observed.

\section{Acknowledgments}

This work was supported by the National Natural Science Foundation of China, under Grants No. 11021092, No. 10975004 and No. 11135003.

[1] G. Jungman, M. Kamionkowski, and K. Griest, Phys.Rept. 267, 195 (1996), hep-ph/9506380.

[2] G. Bertone, D. Hooper, and J. Silk, Phys.Rept. 405, 279 (2005), hep-ph/0404175.

[3] E. Komatsu et al. (WMAP Collaboration), Astrophys.J.Suppl. 192, 18 (2011), 1001.4538.

[4] O. Buchmueller, R. Cavanaugh, D. Colling, A. De Roeck, M. Dolan, et al., Eur.Phys.J. C71, 1722 (2011), 1106.2529 .

[5] S. Profumo, Phys.Rev. D84, 015008 (2011), 1105.5162.

[6] G. Belanger, S. Kraml, and A. Lessa, JHEP 1107, 083 (2011), 1105.4878.

[7] J. Kile and A. Soni, Phys.Rev. D84, 035016 (2011), 1104.5239.

[8] S. Akula, D. Feldman, Z. Liu, P. Nath, and G. Peim, Mod.Phys.Lett. A26, 1521 (2011), 1103.5061.

[9] D. Feldman, K. Freese, P. Nath, B. D. Nelson, and G. Peim, Phys.Rev. D84, 015007 (2011), 1102.2548 .

[10] Y. Bai and H.-C. Cheng, JHEP 06, 021 (2011), 1012.1863.

[11] I. Gogoladze, R. Khalid, Y. Mimura, and Q. Shafi, Phys. Rev. D83, 095007 (2011), 1012.1613.

[12] K. Cheung, K. Mawatari, E. Senaha, P.-Y. Tseng, and T.-C. Yuan, JHEP 10, 081 (2010), 1009.0618 .

[13] J. Goodman et al., Phys. Rev. D82, 116010 (2010), 1008.1783.

[14] G. Bertone, D. G. Cerdeno, M. Fornasa, R. Ruiz de Austri, and R. Trotta, Phys. Rev. D82, 055008 (2010), 1005.4280 .

[15] G. F. Giudice, T. Han, K. Wang, and L.-T. Wang, Phys. Rev. D81, 115011 (2010), 1004.4902. 
[16] T. Li and W. Chao, Nucl. Phys. B843, 396 (2011), 1004.0296.

[17] M. Beltran, D. Hooper, E. W. Kolb, Z. A. C. Krusberg, and T. M. P. Tait, JHEP 09, 037 (2010), 1002.4137.

[18] H. Zhang, C. S. Li, Q.-H. Cao, and Z. Li, Phys. Rev. D82, 075003 (2010), 0910.2831.

[19] N. Arkani-Hamed and N. Weiner, JHEP 12, 104 (2008), 0810.0714.

[20] D. Fargion, M. Y. Khlopov, R. V. Konoplich, and R. Mignani, Phys. Rev. D54, 4684 (1996).

[21] Y. Bai, P. J. Fox, and R. Harnik, JHEP 12, 048 (2010), 1005.3797.

[22] P. J. Fox, R. Harnik, J. Kopp, and Y. Tsai, Phys.Rev. D85, 056011 (2012), 1109.4398.

[23] U. Haisch, F. Kahlhoefer, and J. Unwin (2012), 1208.4605.

[24] S. Chatrchyan et al. (CMS Collaboration), Phys.Rev.Lett. 108, 261803 (2012), 1204.0821.

[25] J. Wang, C. S. Li, D. Y. Shao, and H. Zhang, Phys.Rev. D84, 075011 (2011), 1107.2048.

[26] X. Gao, C. S. Li, J. Gao, J. Wang, and R. J. Oakes, Phys.Rev. D81, 036008 (2010), 0912.0199.

[27] M. Beltran, D. Hooper, E. W. Kolb, and Z. A. C. Krusberg, Phys. Rev. D80, 043509 (2009), 0808.3384.

[28] H. Zhang, Q.-H. Cao, C.-R. Chen, C. S. Li, and JHEP 1108, 018 (2011), 0912.4511.

[29] Y. Mambrini and B. Zaldivar, JCAP 1110, 023 (2011), 1106.4819.

[30] P. J. Fox, R. Harnik, J. Kopp and Y. Tsai, Phys. Rev. D 84, 014028 (2011), 1103.0240.

[31] E. W. Kolb and M. S. Turner, Front.Phys. 69, 1 (1990).

[32] N. Jarosik et al., Astrophys. J. Suppl. 192, 14 (2011), 1001.4744.

[33] B. W. Harris and J. F. Owens, Phys. Rev. D65, 094032 (2002), hep-ph/0102128.

[34] J. Pumplin et al., JHEP 07, 012 (2002), hep-ph/0201195.

[35] T. Bringmann, X. Huang, A. Ibarra, S. Vogl, and C. Weniger, JCAP 1207, 054 (2012), 1203.1312 .

[36] C. Weniger, JCAP 1208, 007 (2012), 1204.2797.

[37] U. Baur and E. L. Berger, Phys. Rev. D47, 4889 (1993).

[38] J. M. Campbell, R. K. Ellis, and C. Williams, JHEP 1107, 018 (2011), 1105.0020.

[39] U. Baur, T. Han, and J. Ohnemus, Phys. Rev. D57, 2823 (1998), hep-ph/9710416.

[40] J. Ohnemus, Phys. Rev. D47, 940 (1993).

[41] W. Giele, E. N. Glover, and D. A. Kosower, Nucl.Phys. B403, 633 (1993), hep-ph/9302225. 